\title{
Radiation Performance of Highly Miniaturized Implantable Devices
}

\author{
Denys Nikolayev ${ }^{* \dagger 1}$, Maxim Zhadobov ${ }^{\ddagger 2}$, Wout Joseph ${ }^{\dagger 3}$, Luc Martens $^{\dagger 4}$, Ronan Sauleau ${ }^{\ddagger 5}$ \\ *Microwaves and Antennas Group (MAG), EPFL, CH-1015 Lausanne, Switzerland \\ 'INTEC - WAVES, imec / Ghent University, BE-9052 Ghent, Belgium \\ *Univ Rennes, CNRS, Institut d'Électronique et de Télécommunications de Rennes, UMR-6164, FR-35000 Rennes, France \\ ${ }^{1} \mathrm{~d} @$ deniq.com, $\left\{{ }^{2}\right.$ Maxim.Zhadobov, ${ }^{5}$ Ronan.Sauleau $\} @$ univ-rennes1.fr, $\left\{{ }^{3}\right.$ Wout.Joseph, ${ }^{4}$ Luc1.Martens $\} @$ ugent.be
}

\begin{abstract}
Miniature wireless implantable bioelectronics provide powerful capabilities for biotelemetry, therapeutics, and neural interfacing. These technologies rely on antennas to communicate with external receivers, yet existing systems suffer from poor radiation performance. We address this issue by studying the through-tissue propagation, deriving the optimal frequency range, and obtaining the maximum achievable farfield radiation efficiency. Three problem formulations are considered with increasing complexity and anatomical realism. Polarization effects of TM and TE modes are investigated using an infinitesimal magnetic dipole and a magnetic current sources, respectively. The optimal operating frequency is found within the $\left[10^{8}, 3 \times 10^{9}\right]-\mathrm{Hz}$ range and can be roughly approximated as $f \approx$ $2.2 \times 10^{7} / d$ for deep implantation (i.e. $d \gtrsim 2 \mathrm{~cm}$ ). Considering the implantation depth, the operating frequency, the polarization, and the directivity, we show that about an order-of-magnitude efficiency improvement is achievable compared to existing devices.
\end{abstract}

Keywords - antenna, bioelectronics, implantable, in-body, ingestible, injectable, phantom, propagation, radiation efficiency.

\section{INTRODUCTION}

In-body bioelectronic devices (Fig. 1) offer an advanced range of capabilities in clinical research, medicine, defense, and professional sports [1]-[3]. For instance, wireless biomedical telemetry allows for monitoring of a wide range of human or animal physiological parameters: vitals, blood analyses, organ monitoring, and so on. Precision medicine requires precision diagnostics [4], and miniature bioelectronics makes it possible to obtain more precise data than ever before about one's health. Neural interfaces allow us to study the brain via mapping, assisting, augmenting, and repairing cognitive or sensory-motor functions [5]. The emerging concept of electroceuticals aims individual neural circuits that regulate the physiological processes to treat a wide range of illnesses [6]. Continuous innovation in microelectromechanical systems (MEMS), integrated circuits (IC), and microfluidics further accelerates the progress in bioelectronics.

Likewise, wireless power transfer (WPT) allows for the removal of bulky batteries and eliminates the need to go through surgery to replace them (Fig. 1). It makes the life span of an implanted device practically unlimited. Efficient theoretical approaches to WPT into tissues have been proposed recently [7]-[9]. Body-conformal transmitarray
Antenna system for wireless data and power transfer

Wirelessly

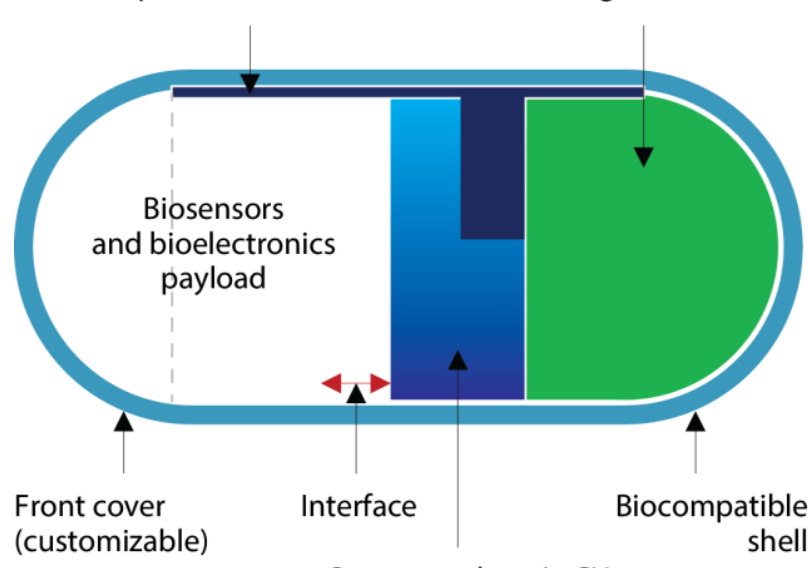

Sensor readout / $\mu \mathrm{CU}$

Fig. 1. Outline of an in-body bioelectronic device that uses a multi-functional antenna for both wireless data and power transfer. Miniaturized antenna, batteries, and circuits are enclosed in a biocompatible shell. Front cover of the device is customizable according to a given application. For instance, a direct interface of a sensor with tissues may be required. Transparent covers are used for ingestible capsule endoscopy.

surfaces can focus energy into tissues [10]. However, efficient radiating structures are required for body-implanted devices to overcome existing limitations on powering, safety, and data transfer [11], [12].

In-body bioelectronics commonly uses radiofrequency (RF) antennas to communicate with external on- or off-body systems. A wide range of RF antennas has been proposed for body-implantable applications [13]-[21]. So far, establishing a robust link between an in-body device and external equipment remains a major challenge because of too low total efficiencies $\left(\eta_{t o t}<0.1 \%\right)$ of the antennas operating in lossy media with uncertain electromagnetic (EM) properties [13]. Considering typical maximum input power levels ranging from a few to about $50 \mathrm{~mW}$ [2] (limited by safety standards) and $\mathrm{Rx}$ sensitivities, this efficiency provides an operating range up to only a few meters [22].

In this study, we 1) describe the mechanisms affecting radiation efficiency in tissues and 2) derive the optimal frequency range. 


\section{Physical Mechanisms Limiting}

\section{THE RADIATION EFFICIENCY OF IMPLANTABLE DEVICES}

From an electromagnetic (EM) perspective, body tissues are an anisotropic, nonlinear, heterogeneous, and dispersive medium [23]. Considering RF frequency range and related power levels, we assume that all EM properties are isotropic (scalar complex permittivity), nonmagnetic $\left(\mu=\mu_{0}\right)$, and linear [no heating of tissues due to low supplied power]. To represent the tissue dispersion, we use the four-region ColeCole model defined in [24] with its coefficients based on experimental data [25]. Fig. 2a shows the EM properties of muscle and fat according to the model. For the majority of tissues, the relative permittivity $\varepsilon_{r} \propto 1 / f$ and conductivity $\sigma \propto f$.

Two main frequency-dependent mechanisms affect the EM energy transfer efficiency from dispersive body tissues to free space [11]: 1) the attenuation due to dielectric and conductive losses, 2) the reflection (mismatch) losses due to waveimpedance contrast. Furthermore, a body and its cavities and organs may exhibit resonance behavior affecting efficiency as well [26]. Related to the antenna itself, the physical limitations of electrically small sources in lossy media limit the radiation efficiency [27]. Note that high permittivity of tissues loads the antenna making its electrical size considerably larger than the physical one [28].

\section{A. Attenuation in Tissues}

For a transverse-electromagnetic (TEM) wave propagating in a lossy environment, the attenuation constant $\alpha$ can be expressed as

$$
\alpha=\omega \sqrt{\mu_{0} \varepsilon} \sqrt{\frac{1}{2}\left[\sqrt{1+\left(\frac{\sigma}{\omega \varepsilon}\right)^{2}}-1\right]},
$$

where $\omega=2 \pi f$.

Alternatively, the penetration depth $\delta_{p}=1 / \alpha$ gives the depth $d$ at which the field amplitude falls by $1 / e$ or the intensity by $1 / e^{2}$. Fig. $2 \mathrm{~b}$ shows the penetration depth $\delta_{p}$ in dispersive muscle and fat tissues. Clearly, this mechanism favors lower frequencies to maximize radiation efficiency.

\section{B. Reflection Losses at Tissue-Air Interface}

The reflection coefficient $\Gamma=E^{-} / E^{+}$(where $E^{-}$is the amplitude of the incident and $E^{+}$of the reflected $\mathbf{E}$-field) depends on wave impedances of two media, $Z_{n}=[(j \omega \mu) /(\sigma+$ $j \omega \varepsilon)]^{-1 / 2}$, where $n=1,2$. As implantable devices radiate mostly from higher to lower permittivity medium (exceptions occur on fat-skin and bone-muscle interfaces, for instance), a significant part of the energy is trapped inside of a body due to the total internal reflection. Assuming the plane wave, for the $s$-(perpendicular) and $p$-polarized (parallel) waves [29],

$$
\begin{gathered}
\Gamma_{s}=\left(Z_{2} \cos \theta_{i}-Z_{1} \cos \theta_{t}\right) /\left(Z_{2} \cos \theta_{i}+Z_{1} \cos \theta_{t}\right), \\
\Gamma_{p}=\left(-Z_{1} \cos \theta_{i}-Z_{2} \cos \theta_{t}\right) /\left(Z_{1} \cos \theta_{i}+Z_{2} \cos \theta_{t}\right),
\end{gathered}
$$

where $\theta_{i}$ is the angle of incidence and $\theta_{i}=\sin ^{-1}\left[\sin \left(\theta_{i}\right) Z_{2} / Z_{1}\right]$ is the angle of refraction.

Fig. 2c and Fig. 2d demonstrate the solution of (2) for $s$ and $p$-polarized plane waves incident upon planar dispersive-

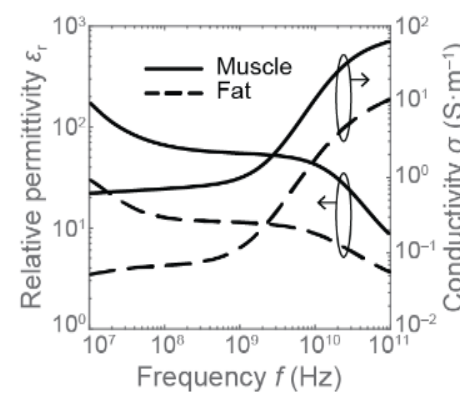

(a)

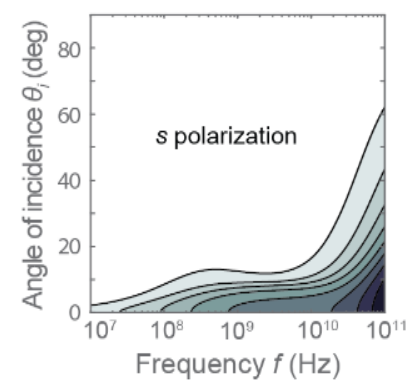

(c)

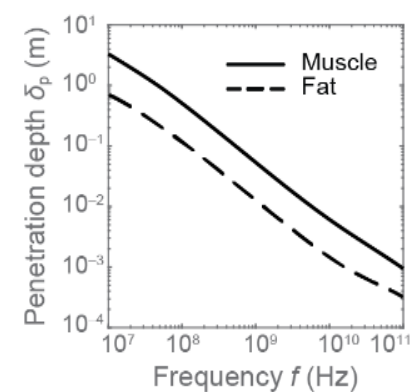

(b)
Fig. 2. Physical mechanisms affecting the radiation efficiency of implantable devices. (a) EM dispersion in muscle and fat modeled using four region ColeCole model based on the data by Gabriel et al. [24]. (b) Penetration depth $\delta_{p}=$ $1 / \alpha$ of a plane wave propagating into dispersive muscle and fat tissues. (d) and (e) Reflection coefficients $|\Gamma|$ of the (d) perpendicular-polarized and (e) parallel-polarized plane waves incident upon planar dispersive-skin-air interface. White area covers the region of total internal reflection. Note also the effect of Brewster's angle for $p$-polarization

skin-air interface. For $\theta_{i}>0, p$-polarization gives better transmission with $\max |\Gamma|=0.5$ at Brewster's angle (Fig. $2 \mathrm{~d}$ ).

In contrast to attenuation in tissues, the reflection losses favor using higher frequencies. As the effect of attenuation increases with the implant depth $d$ in lossy tissues (1), the optimal operating frequency would be inversely proportional to the depth.

\section{Formulation OF THE PROBlem}

We formulate the problem geometry by increasing its complexity from a simple to an anatomically realistic one. First, we consider a planar homogeneous medium consisting of two infinite half-spaces that represent i) free-space domain and ii) tissue domain with muscle-equivalent properties. Next, we add 2-mm skin and 5-mm fat layers to the model as proposed in [30]. Finally, we analyze the heterogeneous realistic model of a human abdominal region (Fig. 3) using the previously developed model [31]. The magnetic dipole and out-of-plane magnetic current line sources are considered [11]. The sources are centered inside a lossless (i.e. $\sigma=0$ ) $\varnothing 4-\mathrm{mm}$ cylindrical area.

The EM field radiated from an arbitrary source in tissues satisfies the inhomogeneous wave equation. In terms of the time-harmonic electric field $\mathbf{E}$ (time variations of the form $\left.e^{j \omega t}\right)$, it is expressed as $\nabla^{2} \mathbf{E}=j \omega \mu \mathbf{J}_{s}+j \omega \mu \sigma \mathbf{E}-\omega^{2} \mu \varepsilon \mathbf{E}$, where $\mathbf{J}_{s}$ is the source electric current density. Assuming $\mathbf{E}(x, y, z)=$ 


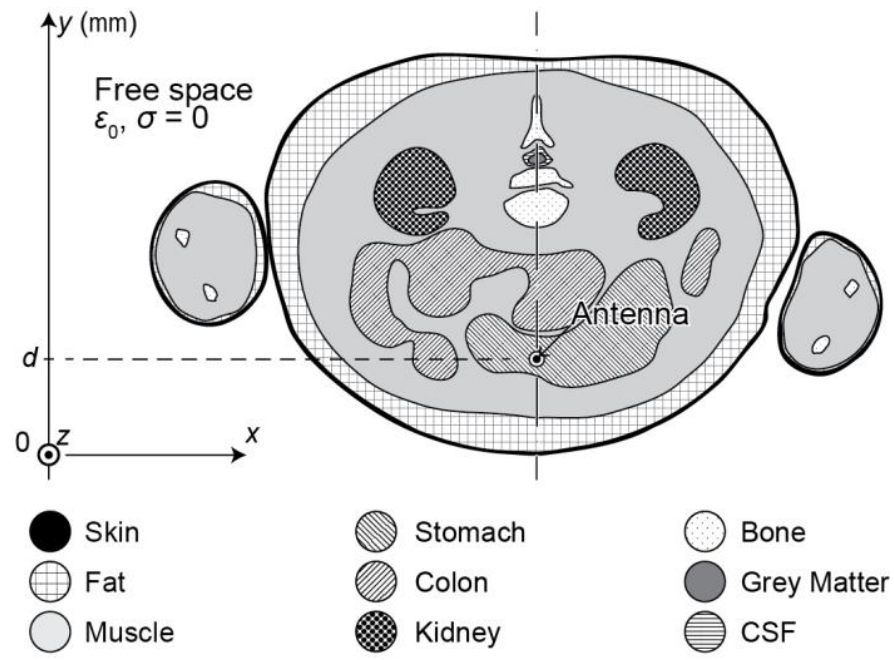

Fig. 3. Problem formulation: heterogeneous nine-tissue realistic model of a human abdominal region (source region is not to scale, $z$ axis is out of plane).

$\mathbf{E}(x, y) e^{i \overline{k z}}$, where $\bar{k}$ is the out-of-plane wave number, we reduce the spatial dimensionality of the problem.

The radiation efficiency $\eta$ is derived from the conservation of energy [29]. Poynting's theorem states that $P_{s}=P_{e}+P_{d}+$ $j 2 \omega\left(\bar{W}_{m}-\bar{W}_{e}\right)$, where

$$
\begin{gathered}
P_{s}=-\frac{1}{2} \int_{\Omega_{S}}\left(\mathbf{H}^{*} \cdot \mathbf{M}_{i}+\mathbf{E} \cdot \mathbf{J}_{i}^{*}\right) d v, \\
P_{e}=\oiiint_{\Sigma_{F F}}\left(\frac{1}{2} \mathbf{E} \times \mathbf{H}^{*}\right) \cdot d s, \\
P_{d}=\frac{1}{2} \oiiint_{\Omega_{P}} \sigma|\mathbf{E}|^{2} d v .
\end{gathered}
$$

In this way, we calculate the radiation efficiency as

$$
\eta \equiv \operatorname{Re}\left(P_{e}\right) / \operatorname{Re}\left(P_{s}\right) \text {. }
$$

The peak of $\eta(\mathrm{f})$ defines the optimal frequency $f_{\text {opt }}$ as $\eta\left(f_{\text {opt }}\right) \equiv \max (\eta)$. Note that for a realistic antenna, the total radiation efficiency also includes mismatch losses as $\eta_{\text {tot }}=$ $\eta\left(1-\left|\Gamma_{A}\right|^{2}\right)$, where $\Gamma_{A}$ is the reflection coefficient at the antenna terminals. In this study, we consider $\Gamma_{A}=0$. Impedance detuning issues of in-body antennas were addressed in [32], [33].

\section{RESULTS}

The analysis in the homogeneous medium revealed that the optimal frequency range is inversely proportional to the depth $d$ of the source in tissues and decays exponentially (Fig. 4). At $d=1 \mathrm{~cm}$, the radiation efficiency maxima are at $480 \mathrm{MHz}$ and $2.1 \mathrm{GHz}$ with a drop in between at $1.3 \mathrm{GHz}$. At the global maximum $(2.1 \mathrm{GHz})$, the destructive interference occurs close to the tissue-air interface, thus reducing the area of dissipated power independently of the source formulation. The position of the local minimum is invariant to the source formulation. As we move deeper in tissues, the optimal frequency shift

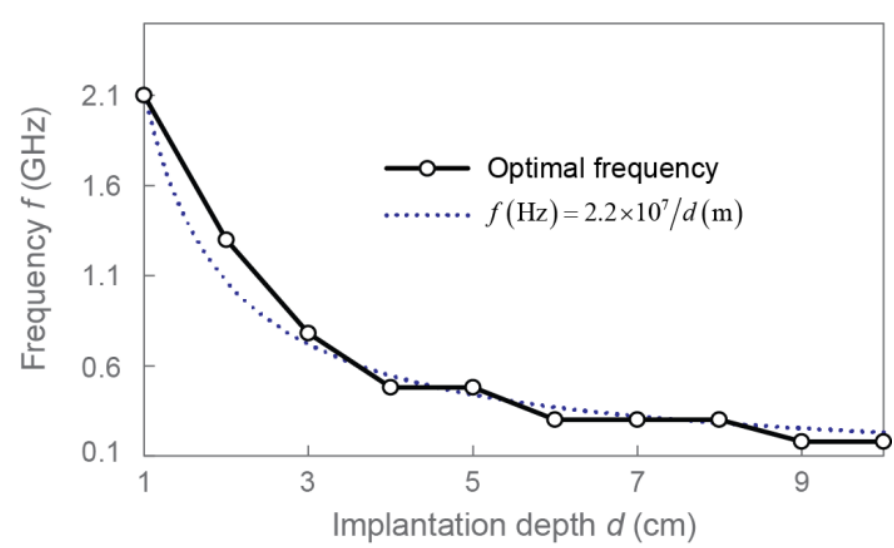

Fig. 4. Optimal frequency of equivalent sources considered in this study is inversely proportional to its depth in tissue.

from about $2 \mathrm{GHz}$ at $1 \mathrm{~cm}$ down to about $200 \mathrm{MHz}$ at $10 \mathrm{~cm}$. Maximum achievable efficiency is about $1.8 \%$.

Adding fat and skin layers do not affect the optimal frequency but modify the achievable efficiency. The fat layer acts as a low-loss matching layer, reducing the reflection losses and thus increasing overall efficiency compared to the homogeneous half-space medium. The efficiency increases by about $50 \%$ at $1 \mathrm{~cm}$ but only $20 \%$ at $6 \mathrm{~cm}$. Using $p$-polarization (magnetic dipole source) improves the efficiency by a few percents (relative increase up to $7 \%$ ), and this effect is inversely proportional to the frequency and depth.

For the anatomical model (Fig. 3), the optimal frequency range for deep implantation $(d \gtrsim 2 \mathrm{~cm})$ corresponds with the canonical models. The maximum achievable efficiency in the anatomical phantom peaks at $16 \%$. Within the optimal frequency range, the $p$-polarization gives relatively about $20 \%$ higher efficiency than the $s$-polarization for all implantation depths due both to higher directivity and lower reflection losses. At a 1-cm depth, the efficiency drops at about $2.1 \mathrm{GHz}$ within the optimal range, independently of the excitation mode, as for the planar homogeneous model. The efficiency at 800 MHz exceeds the one at $2.1 \mathrm{GHz}$ by about 6.5 times. The drop in efficiency is due to constructive interference with the standing surface wave, which increases power absorption in tissues.

\section{CONCLUSION}

Far-field radiative energy transfer from an implantable device to the surrounding free space gives substantially better efficiency than near-field coupling. For an ideal equivalent source, the radiation efficiency increases with frequency until reaching the maximum. The frequencies around this maximum are optimal and inversely proportional to the implantation depth. Maximizing the efficiency for a given depth is negotiating between the attenuation caused the dielectric losses and the wave-impedance mismatch at the tissue-air interface. In addition, for subcutaneous applications, the interference effects induced by standing surface waves are significant. Prior to designing the antenna, considering its implantation depth, operating frequency, mode, and polarization, could result in significant improvement of its radiation efficiency. 


\section{ACKNOWLEDGMENT}

This publication is supported by the European Union through the European Regional Development Fund (ERDF), and by the French region of Brittany, Ministry of Higher Education and Research, Rennes Métropole and Conseil Départemental 35, through the CPER SOPHIE/STIC \& Ondes.

\section{REFERENCES}

[1] A. S. Y. Poon, "Miniaturized Biomedical Implantable Devices," in Implantable Bioelectronics, Weinheim, Germany: Wiley-VCH, 2014, pp. 45-64.

[2] A. Kiourti and K. S. Nikita, "A review of in-body biotelemetry devices: implantables, ingestibles, and injectables," IEEE Trans. Biomed. Eng., vol. 64, no. 7, pp. 1422-1430, Jul. 2017

[3] D. Nikolayev, M. Zhadobov, R. Sauleau, and P. Karban, "Antennas for ingestible capsule telemetry," in Advances in Body-Centric Wireless Communication: Applications and State-of-the-Art, London, UK: IET, 2016, pp. 143-186.

[4] S. J. Aronson and H. L. Rehm, "Building the foundation for genomics in precision medicine," Nature, vol. 526, no. 7573, pp. 336-342, Oct. 2015

[5] J. J. Jun et al., "Fully integrated silicon probes for high-density recording of neural activity," Nature, vol. 551, no. 7679, pp. 232-236, Nov. 2017.

[6] K. Famm, B. Litt, K. J. Tracey, E. S. Boyden, and M. Slaoui, "Drug discovery: A jump-start for electroceuticals," Nature, vol. 496, pp. 159161, Apr. 2013

[7] Y. Urzhumov and D. R. Smith, "Metamaterial-enhanced coupling between magnetic dipoles for efficient wireless power transfer," Phys. Rev. B, vol. 83, no. 20, p. 205114, May 2011.

[8] J. S. Ho, S. Kim, and A. S. Y. Poon, "Midfield wireless powering for implantable systems," Proceedings of the IEEE, vol. 101, no. 6, pp. 1369-1378, Jun. 2013.

[9] A. S. Rekhi and A. Arbabian, "Remote sub-wavelength focusing of ultrasonically activated Lorentz current," Appl. Phys. Lett., vol. 110, no. 16, p. 164104, Apr. 2017

[10] D. R. Agrawal et al., "Conformal phased surfaces for wireless powering of bioelectronic microdevices," Nat. Biomed. Eng., vol. 1, p. 0043, Mar. 2017.

[11] D. Nikolayev, M. Zhadobov, P. Karban, and R. Sauleau, "Electromagnetic radiation efficiency of body-implanted devices," Phys. Rev. Applied, vol. 9, no. 2, p. 024033, Feb. 2018.

[12] A. K. Skrivervik, "Implantable antennas: The challenge of efficiency," in 7th European Conference on Antennas and Propagation (EuCAP 2013), Gothenburg, Sweden, 2013, pp. 3627-3631.

[13] D. Nikolayev, M. Zhadobov, P. Karban, and R. Sauleau, "Conformal antennas for miniature in-body devices: The quest to improve radiation performance," URSI Rad. Sci. Bull., vol. 2017, no. 363, pp. 52-64, Dec. 2017.

[14] Y. Peng, K. Saito, and K. Ito, "Antenna design for impulse-radio-based wireless capsule endoscope communication systems," IEEE Trans. Antennas Propag., vol. 66, no. 10, pp. 5031-5042, Oct. 2018.

[15] S. Bakogianni and S. Koulouridis, "On the design of miniature MedRadio implantable antennas," IEEE Trans. Antennas Propag., vol. 65, no. 7, pp. 3447-3455, Jul. 2017.

[16] D. Nikolayev, M. Zhadobov, L. Le Coq, P. Karban, and R. Sauleau, "Robust ultra-miniature capsule antenna for ingestible and implantable applications," IEEE Trans. Antennas Propag., vol. 65, no. 11, pp. 61076119, Nov. 2017

[17] Z. Bao, Y.-X. Guo, and R. Mittra, "Conformal capsule antenna with reconfigurable radiation pattern for robust communications," IEEE Trans. Antennas Propag., vol. 66, no. 7, pp. 3354-3365, Apr. 2018.

[18] J. Wang, M. Leach, E. G. Lim, Z. Wang, R. Pei, and Y. Huang, "An implantable and conformal antenna for wireless capsule endoscopy," IEEE Antenn. Wireless Propag. Lett., vol. 17, no. 7, pp. 1153-1157, Jul. 2018.

[19] W. Lei and Y. Guo, "Design of a dual-polarized wideband conforma loop antenna for capsule endoscopy systems," IEEE Trans. Antennas Propag., vol. 66, no. 11, pp. 5706-5715, Nov. 2018.

[20] M. M. Suzan, K. Haneda, C. Icheln, A. Khatun, and K. Takizawa, "An ultrawideband conformal loop antenna for ingestible capsule endoscope system," in 10th European Conference on Antennas and Propagation (EuCAP 2016), Davos, Switzerland, 2016, pp. 1-5.

[21] F. Merli, L. Bolomey, J. Zurcher, G. Corradini, E. Meurville, and A. K Skrivervik, "Design, realization and measurements of a miniature antenna for implantable wireless communication systems," IEEE Trans. Antennas Propag., vol. 59, no. 10, pp. 3544-3555, Oct. 2011.

[22] S. Benaissa et al., "Experimental characterization of in-to-out-body path loss at $433 \mathrm{MHz}$ in dairy cows," Electron. Lett., vol. 55, Jan. 2019.

[23] F. S. Barnes and B. Greenebaum, Handbook of biological effects of electromagnetic fields. Bioengineering and biophysical aspects of electromagnetic fields, 3rd ed. Boca Raton: CRC/Taylor \& Francis, 2007.

[24] S. Gabriel, R. W. Lau, and C. Gabriel, "The dielectric properties of biological tissues: III. Parametric models for the dielectric spectrum of tissues," Phys. Med. Biol., vol. 41, pp. 2271-2293, Nov. 1996.

[25] S. Gabriel, R. W. Lau, and C. Gabriel, "The dielectric properties of biological tissues: II. Measurements in the frequency range $10 \mathrm{~Hz}$ to 20 GHz," Phys. Med. Biol., vol. 41, pp. 2251-2269, Nov. 1996.

[26] D. Nikolayev, "Radio frequency resonances inside a human head," Comput. Probl. Electr. Eng., vol. 3, no. 1, pp. 73-78, 2013.

[27] A. Karlsson, "Physical limitations of antennas in a lossy medium," IEEE Trans. Antennas Propag., vol. 52, no. 8, pp. 2027-2033, Aug. 2004.

[28] D. Nikolayev, M. Zhadobov, and R. Sauleau, "Impact of tissue electromagnetic properties on radiation performance of in-body antennas," IEEE Antenn. Wireless Propag. Lett., vol. 17, no. 8, pp. 1440-1444, Aug. 2018.

[29] C. A. Balanis, Advanced Engineering Electromagnetics, Second. Hoboken, NJ: John Wiley \& Sons, 2012

[30] A. S. Y. Poon, S. O'Driscoll, and T. H. Meng, "Optimal frequency for wireless power transmission into dispersive tissue," IEEE Trans. Antennas Propag., vol. 58, no. 5, pp. 1739-1750, May 2010.

[31] D. Nikolayev, "An anatomically realistic boundary representation phantom for studying VHF-UHF radiation effects," Comput. Probl. Electr. Eng., vol. 3, no. 2, pp. 77-82, 2013.

[32] D. Nikolayev, M. Zhadobov, and R. Sauleau, "Immune-to-detuning wireless in-body platform for versatile biotelemetry applications," IEEE Trans. Biomed. Circuits Syst., Jan. 2019.

[33] M. K. Magill, G. A. Conway, and W. G. Scanlon, "Tissue-independent implantable antenna for in-body communications at $2.36-2.5 \mathrm{GHz}$," IEEE Trans. Antennas Propag., vol. 65, no. 9, pp. 4406-4417, Sep. 2017 